\title{
Progressive Impairment on Neuropsychological Tasks in a Longitudinal Study of Preclinical Alzheimer's Disease
}

\author{
Laura Mickes and John T. Wixted \\ University of California, San Diego \\ Douglas Galasko, Mark W. Bondi, and Leon J. Thal \\ University of California, San Diego School of Medicine and \\ Veterans Affairs San Diego Healthcare System
}

\author{
Christine Fennema-Notestine \\ University of California, San Diego School of Medicine \\ David P. Salmon \\ University of California, San Diego School of Medicine
}

\begin{abstract}
Previous research suggests that patients with Alzheimer's disease exhibit cognitive impairment in the years preceding a clinical diagnosis. Memory impairments are particularly pronounced, but the relative degree to which other cognitive functions are impaired and the speed with which they decline during the preclinical years remains unclear. The authors report a detailed neuropsychological evaluation of 11 patients over the course of 3 years up to and including the 1st year of nonnormal diagnosis. The results suggest that performance falls off rapidly in all areas of cognitive functioning but that abilities thought to be subserved by the medial and lateral temporal lobes (episodic and semantic memory, respectively) appear to be substantially more impaired than those abilities thought to be subserved by the frontal lobes.
\end{abstract}

Keywords: longitudinal, mild cognitive impairment, memory, semantic, executive

Alzheimer's disease (AD) produces a progressive dementia that eventually impairs all aspects of cognitive functioning, but its earliest preclinical stages appear to affect memory more than any other cognitive domain. This observation is consistent with prior research showing that the neurodegeneration associated with AD begins in the medial temporal lobes (Braak \& Braak, 1996; Delacourte et al., 1999), an area of the brain known to be important for declarative (and particularly, episodic) memory (Squire \& Zola, 1996). How the disease progresses from this early stage to the more widespread neural degeneration and pervasive pattern of cognitive impairment that characterizes the later stages of $\mathrm{AD}$ is not clear. The purpose of the present investigation is to shed light on this issue by analyzing the neuropsychological profile of 11 preclinical patients. These individuals were among a large group of older adult control subjects who were tested annually as part of a longitudinal neuropsychological investigation of $\mathrm{AD}$. At some

Laura Mickes and John T. Wixted, Department of Psychology, University of California, San Diego; Christine Fennema-Notestine, Department of Psychiatry, University of California, San Diego School of Medicine; Douglas Galasko and Leon J. Thal, Department of Neurosciences, University of California, San Diego School of Medicine, and Veterans Affairs San Diego Healthcare System; Mark W. Bondi, Department of Psychiatry, University of California, San Diego School of Medicine, and Veterans Affairs San Diego Healthcare System; David P. Salmon, Department of Neurosciences, University of California, San Diego School of Medicine.

Support for this study was provided by National Institute on Aging Grants P50 AG05131 and RO1 AG12674 and a Department of Veterans Affairs Medical Research Service REAP Award. We thank Larry Squire, Philip Tseng, Katherine Gardner, and the Alzheimer's Disease Research Center staff and subjects. We are sad to note that Leon J. Thal died February 3, 2007.

Correspondence concerning this article should be addressed to Laura Mickes, Department of Psychology, University of California, San Diego, 9500 Gilman Drive, La Jolla, CA 92093-0109. E-mail: 1mickes@ ucsd.edu point, these control subjects were diagnosed with probable $\mathrm{AD}$, and their performance on a variety of standard neuropsychological tests during the 2 years preceding a nonnormal diagnosis reflects a rapid decline in various aspects of their cognitive functioning. The relative rates of decline on these tests may provide an indication of how $\mathrm{AD}$ progresses in the brain after its onset in the medial temporal lobes.

Prior research on the progression of preclinical AD yields a mixed picture. In recent reviews of the relevant literature, Backman, Jones, Berger, Laukka, and Small $(2004,2005)$ argued that the cognitive decline in the 2 or 3 years preceding a nonnormal diagnosis is largely nonspecific. In fact, according to their reading of the literature, even the widely accepted idea that memory is differentially impaired in preclinical AD patients may be overstated. They observed that although memory was clearly impaired, so were executive functioning, perceptual speed, verbal ability, visuospatial skill, and attention. This broad-based decline in cognitive functioning mirrors evidence suggesting that multiple brain structures are impaired in preclinical $\mathrm{AD}$, structures that include (but are not limited to) the medial temporal lobes, the frontal lobes, and the anterior cingulate (Albert, Moss, Tanzi, \& Jones, 2001; Small, Mobly, Laukka, Jones, \& Backman, 2003).

However, the definition of what constitutes the preclinical stage differs across the various studies reviewed in the Backman et al. (2005) meta-analysis. Some studies involved cognitively normal individuals who later converted to $\mathrm{AD}$, whereas others involved patients with mild cognitive impairment (MCI) who later converted to $\mathrm{AD}$. In addition, some studies measured the degree of deficit in various domains of cognitive functioning, whereas others used neuropsychological measures to predict who among a group of cognitively normal individuals (or a group of MCI patients) would convert to AD. Finally, control groups in many of the studies were not matched with respect to important variables, such as age (with converters frequently being older than controls) and education. In fact, matching is not even a goal of prediction 
studies, especially community-based studies in search of possible screening tests for AD. By design, these studies ask how converters in a large sample differ from those who remained cognitively stable (not how they differ from a matched control group; e.g., see Saxton et al., 2004).

The present study was designed to evaluate the neuropsychological performance of presumptively normal individuals who ultimately converted to a diagnosis of probable $\mathrm{AD}$ relative to that of a carefully matched control group. The goal was to quantify the degree of impairment in episodic memory, semantic knowledge, and executive functioning in the 2 years preceding a nonnormal diagnosis in an effort to shed light on the neuroanatomical progression of preclinical AD. To that end, we report control-referenced $z$-scores for the performance of these patients on the California Verbal Learning Test (CVLT; Delis, Kramer, Kaplan, \& Ober, 1987), the modified Wisconsin Card Sort Test (mWCST; Nelson, 1976; cf. Lineweaver, Bondi, Thomas, \& Salmon, 1999), the Trail Making Test-Parts A and B (TMT-A and TMT-B; from the Halstead Reitan Neuropsychological Test Battery; see Reitan, 1958), letter and category verbal fluency tests (Butters, Granholm, Salmon, Grant, \& Wolfe, 1987), and a modified version of the Boston Naming Test (BNT; Kaplan, Goodglass, \& Weintraub, 1983). We report these scores for the 2 years preceding a nonnormal diagnosis and for the year in which that diagnosis was made.

\section{Correlating Neuropsychological Test Performance With Brain Pathology}

The pattern of deficits on these neuropsychological tests may shed light on how the neurodegeneration associated with $\mathrm{AD}$ advances in the brain from its presumed starting point in the medial temporal lobes, but complications arise because tests that are purported to be differentially sensitive to the integrity of specific regions of the brain are often found to be equally affected by damage to other parts of the brain as well. For example, the Wisconsin Card Sorting Test (WCST; Heaton, Chelune, Talley, Kay, \& Curtis, 1993) is a standard measure of frontal integrity, but its specificity to that region of the brain is often questioned, because some studies have shown that the integrity of other brain regions correlates with performance on the test as well (e.g., Anderson, Damasio, Jones, \& Tranel, 1991).

Recent meta-analyses, which aggregate effect sizes across a large number of studies, have helped to sort out this complicated literature. For example, Demakis (2003) recently showed that the WCST actually is differentially sensitive to the presence or absence of frontal lobe impairment (just as it is usually assumed to be). This analysis squares with the results of neuroimaging studies that have reported differential activation of the frontal lobes during WCST performance (e.g., Berman et al., 1995). In another metaanalysis, Demakis (2004) found that the TMT-A was differentially sensitive to frontal versus nonfrontal damage. The TMT-B was as well, but to a lesser and (surprisingly) nonsignificant extent. Still, the effect was in the right direction and was nearly significant, so we include the TMT-B in our frontal battery.

Another recent meta-analysis, by Henry, Crawford, and Phillips (2004), suggested that performance on the letter (or phonemic) verbal fluency test, like performance on the TMT-A and the WCST, is differentially sensitive to frontal impairment. By contrast, performance on the category (or semantic) verbal fluency test was affected by damage to either the frontal lobes or to the lateral temporal lobes. This result makes sense because successful performance on this test depends on semantic knowledge, and semantic knowledge is generally thought to be subserved by the lateral temporal lobes (e.g., Levy, Bayley, \& Squire, 2004). In a separate meta-analysis that is highly relevant to our current investigation, Henry et al. found that patients with AD exhibit a more pronounced impairment on the category verbal fluency test than on the letter verbal fluency test, which suggests a differential deficit in the storage of semantic knowledge. This conclusion was bolstered by the observation that $\mathrm{AD}$ patients also exhibited a differential impairment on another test of semantic knowledge, the Boston Naming Test (cf. Monsch et al., 1994).

Thus, on balance, these results raise the possibility that the progression of $\mathrm{AD}$ in the brain is from the medial temporal to the lateral temporal to the frontal lobes. If so, one might expect to find that, in preclinical patients, memory impairment is followed by impairments in semantic knowledge and, only later, by impairments on tests of executive functioning that are relatively specific to frontal lobe integrity (e.g., TMT-A and TMT-B, WCST, and letter fluency). At the other extreme, impairment in preclinical AD patients may exhibit a relatively nonspecific pattern involving similar deficits on the entire battery of neuropsychological tests (cf. Backman et al., 2005).

To maximize power to detect differences in the degree of impairment across different domains of cognitive functioning, we report analyses of tests that are grouped in a conceptually meaningful way. The grouping is partly based on the recent metaanalyses reviewed above and partly on what is known about the brain basis of episodic memory. Thus, we derived an aggregate measure of episodic memory (theoretically, an index of medial temporal lobe integrity) by averaging patient $z$-scores for three memory indices from the CVLT (Delis et al., 1987). An aggregate measure of executive functioning (theoretically, an index of frontal lobe integrity) was derived by averaging the patient $z$-scores on the mWCST, the letter verbal fluency test, and TMT-A and TMT-B. Finally, an aggregate measure of semantic knowledge (theoretically, an index of lateral temporal lobe integrity) was derived by averaging $z$-scores from the BNT and the category verbal fluency test.

\section{Method}

\section{Subjects}

To identify presumptively normal subjects who later converted to $\mathrm{AD}$ (CONVs), we screened existing data from normal control (NC) subjects who were enrolled in an ongoing study at the University of California, San Diego (UCSD) Alzheimer's Disease Research Center (ADRC) from 1987 to 2005. Data from control subjects who at some point had had an annual evaluation in which probable $\mathrm{AD}$ was the consensus diagnosis were reviewed further.

Individuals were excluded from further consideration if they had medical conditions that could potentially cause dementia (e.g., hypothyroidism, vitamin B12 deficiency, electrolyte imbalance), a history of severe head injury, alcoholism, or serious psychiatric disturbance. After taking into account these exclusion criteria, we identified 25 control subjects who eventually converted to a diagnosis of probable AD. Clinical diagnoses were made by two ADRC staff neurologists following the National Institute of Neu- 
rological and Communicative Diseases and Stroke-Alzheimer's Disease and Related Disorders Association guidelines (McKhann et al., 1984). Ten CONVs were excluded from further analysis, 1 due to unusually early onset (32 years old at time of conversion), 2 because they refused to complete the CVLT, and 7 because they were seen prior to the inclusion of the measures of interest (i.e., CVLT) in the ADRC battery of tests.

Although all 15 of the remaining subjects eventually converted to a diagnosis of probable $\mathrm{AD}$, for purposes of analysis we designated the 1st year in which a nonnormal diagnosis was given as the year of conversion (henceforth referred to as "year $n$ "). That is, year $n$ was used to mark the first definite signs of clinical impairment, whether that involved a diagnosis of mild cognitive impairment (MCI; see Petersen, 2004), possible AD, or probable AD. We then examined the neuropsychological profile 1 year (year $n-1$ ) and 2 years (year $n-$ 2) prior to the first year of nonnormal diagnosis.

The diagnostic criteria for MCI were not proposed before 1999. Thus, to ensure that none of the 15 identified CONVs were cognitively impaired in the years preceding their clinical diagnosis, a neurologist and two neuropsychologists jointly rereviewed their pre-1999 records. The histories were reviewed with particular attention to the presence of subjective memory complaints, ratings of functional abilities (assessed by the Functional Activities Questionnaire), overall cognitive performance indexed by the MiniMental State Examination (MMSE; Folstein, Folstein, \& McHugh, 1975), presence of memory impairment (performance worse than 1.5 standard deviations below the age- and education-corrected norms on the Logical Memory Test, a subtest of the Wechsler, 1945, Memory Scale, or on the CVLT), and absence of significant deficits in other cognitive domains. At each visit, subjects were rediagnosed as either controls, as having MCI (following the criteria for amnestic MCI proposed by Petersen, 2004), or as having dementia. This procedure led to the removal of 4 additional subjects who had been diagnosed as normal controls before the introduction of MCI as a clinical entity. Thus, 11 CONVs were included in the study. Of these, 9 received a diagnosis of MCI in year $n$ (and later progressed to probable AD), and 2 received a diagnosis of probable AD in year $n$. Seven of the 11 CONVs subsequently died, and all had pathological diagnoses of definite $\mathrm{AD}$, confirming the clinical diagnoses (overall clinical diagnoses match the pathological diagnoses at $90 \%$; $\mathrm{Ga}-$ lasko et al., 1994; Gearing et al., 1995). Diagnostic criteria for amnestic MCI are that the person must have a subjective memory complaint, low scores on objective memory tests, and relatively preserved daily functioning. None of the CONVs met these criteria in years $n-2$ or $n-1$. Although several CONVs scored 1.5 standard deviations below the mean of the control group on a particular cognitive test in years $n-2$ or $n-1$, no CONV had subjective cognitive complaints that would support a diagnosis of MCI or dementia at that time.

For comparison purposes, eleven NC subjects were identified who matched the $11 \mathrm{CONVs}$ on age, years of education, and years of participation from the same retrospective data set in the ADRC longitudinal study. All NCs retained normal status across all ADRC visits to date.

Table 1 shows the mean age, years of education, MMSE score, and the Dementia Rating Scale score (DRS; Mattis, 1976) for the NCs and CONVs for the 2 years prior to and for the year of conversion. During the year in which the CONVs received nonnormal diagnosis, groups did not differ significantly on mean age, $t(10)=0.13, p=.90$, or mean years of education, $t(10)=0.0$, $p=1.00$. CONVs scored significantly lower than the NC subjects on the two measures of overall cognitive impairment and dementia severity: MMSE, $t(20)=2.14 ; p=.05$, and the DRS, $t(20)=4.62 ; p=.01$. Note that MMSE and DRS scores were within normal limits for all subjects prior to conversion.

\section{Measures}

Psychometrists administered an extensive battery of neuropsychological tests to each subject individually in a quiet and well-lit room on each visit. Administration of the test battery lasted approximately $3 \mathrm{hr}$. The measures selected for examination in the present study are described below. They were selected because they are the measures from the domains of episodic memory, semantic knowledge, and executive functioning that are most sensitive to early AD in the University of California, San Diego ADRC test battery (see Salmon et al., 2002).

MMSE:

This brief, standardized measure assesses cognitive status by examining recall, orientation, attention, language, and the ability to follow simple verbal and written instructions. Scores on this measure range from 0 to 30 , with 30 reflecting perfect performance.

Table 1

Normal Control (NC) and Converter (CONV) Characteristics Across all 3 Years

\begin{tabular}{|c|c|c|c|c|c|c|}
\hline \multirow[b]{2}{*}{ Variable } & \multicolumn{2}{|c|}{$n-2$} & \multicolumn{2}{|c|}{$n-1$} & \multicolumn{2}{|c|}{$n$} \\
\hline & $\mathrm{NC}$ & CONV & $\mathrm{NC}$ & $\mathrm{CONV}$ & $\mathrm{NC}$ & $\mathrm{CONV}$ \\
\hline No. of subjects & 11 & 11 & 11 & 11 & 11 & 11 \\
\hline Age in years & $77.3(5.9)$ & $77.1(4.4)$ & $77.7(6.1)$ & $78.0(4.5)$ & $78.8(6.4)$ & $78.9(4.7)$ \\
\hline Education in years & $16.9(2.0)$ & $16.9(2.8)$ & $16.9(2.0)$ & $16.9(2.8)$ & $16.9(2.0)$ & $16.9(2.8)$ \\
\hline MMSE & $29.6(0.7)$ & $28.1(2.2)$ & $29.6(0.7)$ & $27.7^{*}(1.6)$ & $29.1(1.0)$ & $27.6^{*}(2.2)$ \\
\hline DRS & $139.3(4.1)$ & $136.3(5.3)$ & $139.6(3.5)$ & $134.5^{*}(5.2)$ & $139.6(3.4)$ & $128.8^{*}(6.9)$ \\
\hline
\end{tabular}

Note. Data represent means (and standard deviations). $n-2=2$ years before conversion; $n-1=1$ year before conversion; $n=$ year of conversion. $\mathrm{NC}=$ normal control subjects; CONV = subjects whose diagnosis converted to Alzheimer's disease during the study; MMSE = Mini-Mental State Examination; DRS $=$ Dementia Rating Scale.

${ }^{*} p<.05$. 
DRS:

CVLT:

mWCST:

$B N T$ :

TMT-A and TMT-B: The measures of interest are the time to complete Part A (150-s maximum) and Part B (300-s maximum).
Results

Table 2 shows the raw scores for the CONVs and the NCs for each neuropsychological measure for years $n-2, n-1$, and $n$ ( $n$ -2 is 2 years before conversion, $n-1$ is 1 year before conversion, and $n$ is the year of conversion). Some of the free recall data for the CONVs presented in Table 2 were previously analyzed by Lange et al. (2002) as part of an unrelated investigation of the effect of apolipoprotein E (ApoE) genotype on verbal memory. Unless otherwise noted, an alpha level of .05 was used throughout. For repeated measures tests, the Huyn-Feldt correction was used whenever epsilon was less than .90 .

The main focus of our analysis was on differences in the degree of deficit exhibited by the CONVs relative to that of the matched controls on the various measures of cognitive functioning. To increase power to detect differences, we averaged conceptually similar test scores together into aggregate measures of episodic memory, semantic knowledge, and executive functioning. However, this cannot be done directly because the various scores are measured on different scales (e.g., recognition is measured as $d^{\prime}$ whereas the two recall measures are measured as percent correct). One way to address that problem is to transform the CONV scores to $z$-scores on the basis of the performance of the NCs (e.g., Yonelinas et al., 2002). Doing so places all of the measures on a common scale. Because the CONV $z$-scores are based on the mean

Table 2

Means, (SDs), and Range for Measures of Executive Functioning, Semantic Knowledge, and Episodic Memory

\begin{tabular}{|c|c|c|c|c|c|c|}
\hline \multirow[b]{2}{*}{ Measure } & \multicolumn{2}{|c|}{$n-2$} & \multicolumn{2}{|c|}{$n-1$} & \multicolumn{2}{|c|}{$n$} \\
\hline & $\mathrm{NC}$ & CONV & $\mathrm{NC}$ & CONV & $\mathrm{NC}$ & CONV \\
\hline \multicolumn{7}{|c|}{ Executive functioning } \\
\hline mWCST (perseverative errors) & $\begin{array}{c}0(0) \\
0\end{array}$ & $\begin{array}{c}0.6(1.2) \\
0-3.0\end{array}$ & $\begin{array}{c}0.7(1.4) \\
0-4.0\end{array}$ & $\begin{array}{c}1.0(1.5) \\
0-4.0\end{array}$ & $\begin{array}{c}0.4(1.2) \\
0-4.0\end{array}$ & $\begin{array}{c}1.6(3.2) \\
0-8.0\end{array}$ \\
\hline Letter Fluency (number of correct responses) & $\begin{array}{c}43.0(10.2) \\
29.0-58.0\end{array}$ & $\begin{array}{l}42.9(5.5) \\
36.0-51.0\end{array}$ & $\begin{array}{c}41.9(14.3) \\
26.0-65.0\end{array}$ & $\begin{array}{l}37.7(6.6) \\
31.0-51.0\end{array}$ & $\begin{array}{c}42.1(12.9) \\
22.0-66.0\end{array}$ & $\begin{array}{l}36.7(7.4) \\
24.0-49.0\end{array}$ \\
\hline TMT-A (seconds) & $\begin{array}{c}47.8(21.2) \\
28.0-95.0\end{array}$ & $\begin{array}{l}48.4(9.5) \\
31.0-65.0\end{array}$ & $\begin{array}{c}41.2(10.3) \\
27.0-61.0\end{array}$ & $\begin{array}{c}52.0^{*}(13.3) \\
30.0-77.0\end{array}$ & $\begin{array}{c}40.3(13.0) \\
22.0-63.0\end{array}$ & $\begin{array}{c}60.7^{*}(12.5) \\
43.0-88.0\end{array}$ \\
\hline TMT-B (seconds) & $\begin{array}{l}94.8(36.3) \\
57.0-164.0\end{array}$ & $\begin{array}{c}125.5(32.4) \\
92.0-180.0\end{array}$ & $\begin{array}{l}91.1(28.6) \\
50.0-152.0\end{array}$ & $\begin{array}{c}150.2^{*}(58.4) \\
85.0-300.0\end{array}$ & $\begin{array}{l}97.3(39.7) \\
54.0-190.0\end{array}$ & $\begin{array}{c}171.4^{*}(61.6) \\
82.0-300.0\end{array}$ \\
\hline \multicolumn{7}{|c|}{ Semantic knowledge } \\
\hline $\begin{array}{l}\text { Category Fluency (number of correct responses) } \\
\text { BNT (number of correct responses) }\end{array}$ & $\begin{array}{c}49.1(10.1) \\
38.0-74.0 \\
28.7(1.6) \\
25.0-30.0\end{array}$ & $\begin{array}{c}36.1^{*}(6.0) \\
26.0-46.0 \\
24.9^{*}(3.6) \\
18.0-30.0\end{array}$ & $\begin{array}{l}47.5(6.9) \\
41.0-63.0 \\
27.8(2.4) \\
24.0-30.0\end{array}$ & $\begin{array}{c}32.3^{*}(4.8) \\
23.0-40.0 \\
24.4^{*}(3.2) \\
20.0-29.0\end{array}$ & $\begin{array}{l}45.7(9.3) \\
36.0-70.0 \\
28.1(1.9) \\
24.0-30.0\end{array}$ & $\begin{array}{c}30.4^{*}(9.4) \\
16.0-48.0 \\
23.5^{*}(4.1) \\
15.0-29.0\end{array}$ \\
\hline \multicolumn{7}{|c|}{ Episodic memory $^{\mathrm{a}}$} \\
\hline Recognition score $\left(\mathrm{d}^{\prime}\right)$ & $\begin{array}{l}3.0(.6) \\
2.3-4.0\end{array}$ & $\begin{array}{l}2.4(.9) \\
1.1-4.0\end{array}$ & $\begin{array}{l}3.1(.5) \\
2.5-3.9\end{array}$ & $\begin{array}{l}2.5(.9) \\
1.4-3.9\end{array}$ & $\begin{array}{l}3.2(.5) \\
2.6-4.0\end{array}$ & $\begin{array}{c}2.4^{*}(.5) \\
1.6-3.3\end{array}$ \\
\hline Free recall score & $\begin{array}{c}11.4(3.7) \\
6.0-16.0\end{array}$ & $\begin{array}{c}6.8^{*}(2.7) \\
4.0-10.0\end{array}$ & $\begin{array}{c}11.6(3.6) \\
6.0-16.0\end{array}$ & $\begin{array}{c}4.6^{*}(3.3) \\
1.0-11.0\end{array}$ & $\begin{array}{c}11.4(2.9) \\
5.0-16.0\end{array}$ & $\begin{array}{c}4.7^{*}(3.4) \\
0.0-8.0\end{array}$ \\
\hline Cued recall score & $\begin{array}{c}11.8(3.7) \\
5.0-16.0\end{array}$ & $\begin{array}{l}8.4^{*}(1.6) \\
6.0-11.0\end{array}$ & $\begin{array}{c}11.7(3.1) \\
7.0-16.0\end{array}$ & $\begin{array}{l}6.0^{*}(3.2) \\
2.0-14.0\end{array}$ & $\begin{array}{l}11.6(2.7) \\
7.0-16.0\end{array}$ & $\begin{array}{l}5.8^{*}(2.6) \\
2.0-10.0\end{array}$ \\
\hline
\end{tabular}

Note. $\quad n-2=2$ years before conversion; $n-1=1$ year before conversion; $n=$ year of conversion; $\mathrm{NC}=$ normal control subjects; CONV $=$ subjects whose diagnosis converted to Alzheimer's disease during the study; mWCST = Modified Wisconsin Card Sorting Test; TMT-A = Trail Making Test-Part A; TMT-B $=$ Trail Making Test-Part B; BNT = Boston Naming Test.

${ }^{\text {a }}$ Scores from the California Verbal Learning Test.

${ }^{*} p<.05$. 
and standard deviation of the NC raw scores, it is important to first examine those raw scores in more detail before considering the results of the $z$-score analysis.

\section{Control (NC) Raw Scores}

The raw scores for the NC group on the three episodic memory tests (free recall, cued recall, and recognition) from the CVLT for years $n-2, n-1$, and $n$ were examined. The recall scores reflect the proportion of correct responses on each test, and the recognition score is $d^{\prime}$. In the NC group, performance on these tests did not change in any appreciable way over the 3 years of interest. That is, neither the mean score nor the standard deviation exhibited any systematic trend. A separate one-way analysis of variance (ANOVA) was performed on each dependent measure, and none of the tests approached significance. The tests of executive functioning show a similar level of stability. Performance on the mWCST-Perseverative Errors, letter fluency test, TMT-A and TMT-B remained essentially constant over the 3 years, and separate one-way ANOVAs performed on each measure yielded results that were all far from significant. Similarly, no significant trends in performance were evident over the 3 years for the tests of semantic knowledge.

Because no trends were evident over the 3 years of interest, the $\mathrm{NC}$ data for each dependent measure were aggregated over years to produce one mean and one standard deviation for each measure, which were then used to compute control-referenced $z$-scores for the CONV data. Thus, for example, the mean free recall score for the NC group was obtained by taking the average free recall score over all 3 years, and the pooled standard deviation was obtained by taking the square root of the average variance of the free recall scores over all 3 years. CONV $z$-scores were then computed for years $n-2, n-1$, and $n$ separately as follows: $z_{\mathrm{i}}=\left(s_{\mathrm{i}}-\mu\right) / \sigma$ where $z_{\mathrm{i}}$ is the CONV's $z$-score, $s_{\mathrm{i}}$ is the CONV's individual score (e.g., the raw score on the free recall test for year $n-2$ ), $\mu$ is the corresponding mean score of the NC group on the corresponding test (e.g., the mean recall score across all 3 years), and $\sigma$ is the standard deviation of those scores (pooled over all 3 years).

The conversion of patient raw score to control referenced $z$ scores is potentially complicated by the fact that any anomaly in the distribution of raw scores for the controls can distort the $z$-scores for all of the patients (e.g., Wixted \& Squire, 2004). Thus, before converting the patient scores to $z$-scores, the distributions of raw scores for the control subjects on each test were visually inspected for evidence of outliers. As none were apparent, the $z$-scores were subjected to further statistical and theoretical analysis.

\section{CONV z-Score Analyses}

Preliminary analyses. Table 3 presents the mean CONV zscores (and standard deviations) for each test for years $n-2, n-$ 1 , and $n$. The TMT-A and TMT-B $z$-scores were actually positive (i.e., patients were generally slower than controls), but they are shown as negative scores so that negative values in the table always indicate a deficit in performance. With the exception of letter fluency scores in year $n-2$, all of the $z$-scores are negative. Some of the deficits in years $n-2$ and $n-1$ seem large for subjects who had not yet received a nonnormal diagnosis, but it is important to keep in mind that these scores are relative to a high-functioning matched control group. Relative to standard norms, the deficits are less conspicuous. For example, on the Logical Memory Test, which was used in the diagnostic evaluation, the mean age-corrected scale scores for the control group were above normal for years $n-2, n-1$, and $n(M=13.3,12.8$, and 13.7 , respectively), whereas the corresponding scores for the CONVs were in the normal range for years $n-2$ and $n-1(M \mathrm{~s}$ $=10.3$ and 10.8 , respectively) and fell below normal only in year $n(M=8.8)$.

The $z$-scores shown in Table 3 decline over time, but the

Table 3

Means (SDs) of Control-Referenced z-Scores for CONV on Measures of Executive Functioning, Semantic Knowledge, and Episodic Memory

\begin{tabular}{lccc}
\hline \multicolumn{1}{c}{ Measure } & $n-2$ & $n-1$ & $n$ \\
\hline & Executive functioning & & \\
mWCST (perseverative errors) & $-0.19(1.2)$ & $-0.64(1.5)$ & $-1.28(3.2)$ \\
Letter fluency & $0.05(0.5)$ & $-0.38(0.5)$ & $-0.46(0.6)$ \\
TMT-A & $-0.34(0.6)$ & $-0.58(0.9)$ & $-1.15(0.8)$ \\
TMT-B & $-1.29(1.3)$ & $-1.63(1.7)$ & $-1.80(2.3)$ \\
& & & \\
& Semantic knowledge & & $-1.98(1.1)$ \\
Category Fluency & $-1.31(0.7)$ & $-1.76(0.6)$ & $-2.48(2.2)$ \\
BNT & $-1.72(1.9)$ & $-2.00(1.8)$ & $-1.39(1.0)$ \\
& & & $-1.89(0.8)$ \\
Recognition & Episodic memory & & $-2.02(1.0)$ \\
Cued Recall & $-1.34(1.7)$ & $-1.09(1.7)$ & $-1.83(1.0)$ \\
Free Recall & $-1.08(0.5)$ & $-2.08(1.0)$ & \\
\hline
\end{tabular}

Note. $\quad \mathrm{CONV}=$ subjects whose diagnosis converted to Alzheimer's disease during the study; $n-2=2$ years before conversion; $n-1=1$ year before conversion; $n=$ year of conversion; $\mathrm{mWCST}=$ Modified Wisconsin Card Sorting Test; TMT-A $=$ Trail Making Test-Part A; TMT-B = Trail Making Test-Part B; BNT = Boston Naming Test. 
recognition scores are an exception in that they exhibit substantial impairment in year $n-2$ but no further impairment over the next 2 years. Despite that apparent trend, a repeated measures ANOVA with type of memory test (recall, recognition, and cued recall) and time (years $n-2, n-1$, and $n$ ) as within-participants factors did not yield a significant interaction. Thus, we averaged these three measures into a single aggregate measure of episodic memory.

A similar ANOVA performed on the semantic knowledge measures (BNT and category fluency) also yielded no significant effects involving the type of test. Thus, the average of these two measures yielded an aggregate measure of semantic knowledge. Finally, an ANOVA was performed on the measures of executive functioning (mWCST, letter fluency, TMT-A, and TMT-B). The main effect of type of test was marginally significant, owing mainly to the more pronounced impairment on TMT-B relative to the other measures, $F(2.291,22.91)=2.491, p<.10$, but the interaction between test type and time did not approach significance. Thus, an aggregate measure of executive functioning was also created from these measures.

Aggregate analyses. Figure 1 shows the aggregate $z$-score values for each cognitive domain (episodic memory, semantic knowledge, and executive functioning). The results suggest that performance on tests that correlate with frontal integrity is less impaired than performance on tests that are sensitive to the integrity of the temporal lobes (whether medial or lateral). An ANOVA performed on the data from the preclinical years revealed a significant effect of test type, $F(2,20)=5.33, p<.05$, a significant effect of time, $F(1,10)=5.79, p<.05$, and no interaction. A follow-up comparison between the semantic scores and executive scores yielded a main effect of test, $F(1,10)=14.90, p<.05$, as did a comparison between the episodic memory scores and executive functioning scores, $F(1,10)=5.48, p<.05$. By contrast, the difference between the episodic memory and semantic knowledge measures did not approach significance. Thus, performance on all measures declined at a similar rate during the preclinical years, but the episodic memory and semantic knowledge measures, although not differing from each other, were substantially more impaired than the measures of executive functioning.

Figure 2 presents a more detailed depiction of the data that are summarized in Figure 1. The figure shows the CONV $z$-score distribution for each aggregate measure for years $n-2, n-1$, and $n$ (each symbol represents the score for $1 \mathrm{CONV}$ ). These data confirm the trends that are evident in the averaged data. Thus, for example, for the executive measures, only a few CONVs have $z$-scores less than -1.0 in the preclinical years. By contrast, a high percentage of CONVs have $z$-scores less than -1.0 for the episodic memory and semantic knowledge measures. Thus, the average episodic memory and semantic knowledge $z$-scores shown in Figure 1 are not due to a few outliers having a disproportionate effect. Instead, the mean values appear to be representative of the individual CONV scores. For the executive measure, most of the CONVs have scores less than -1.0 even in year $n$, though 2 patients have $z$-scores that are substantially below the scores of the other CONVs in that year. The impaired score on the aggregate measure of executive performance for these 2 patients mainly reflects the large number of perseverative errors they made on the mWCST.

Regarding the number of perseverative errors made by each CONV for years $n-2, n-1$, and $n$, the large majority of CONVs made zero errors in all 3 years. However, 2 CONVs exhibited a pronounced deterioration in performance on this measure such that

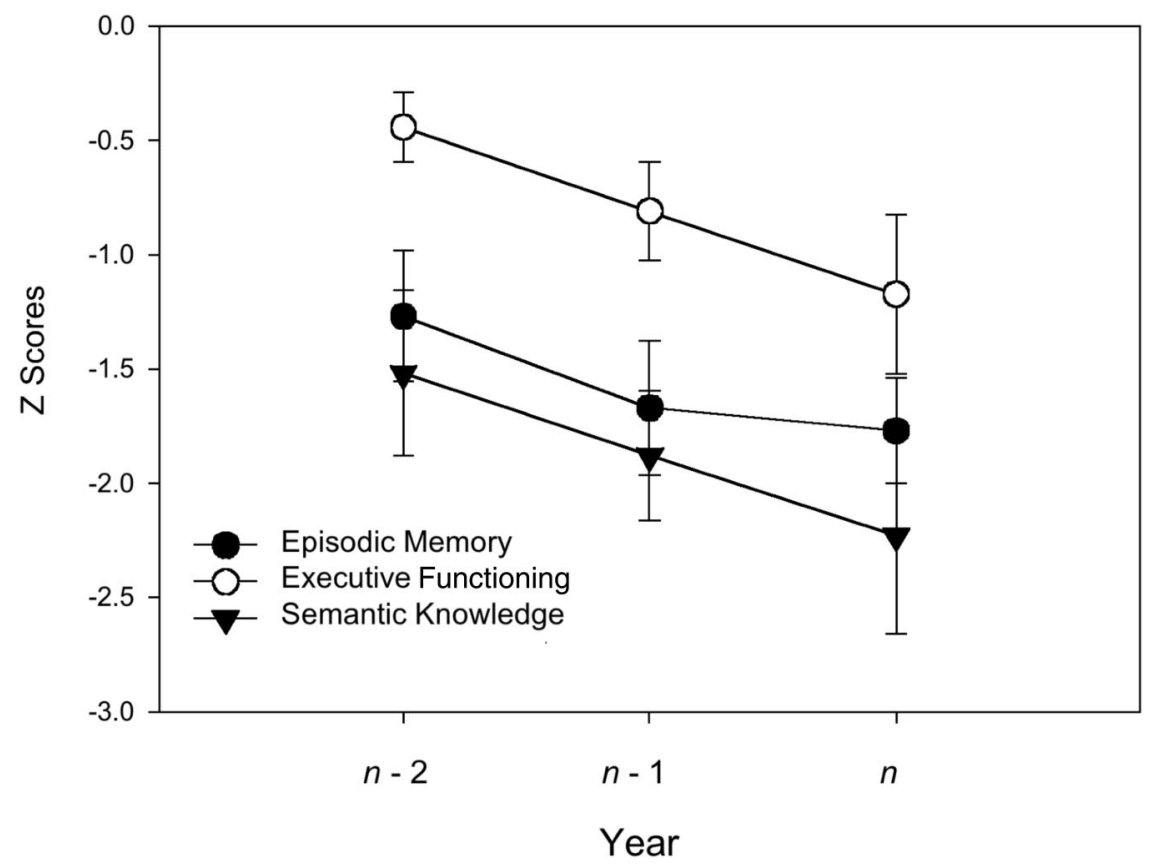

Figure 1. Aggregate control-referenced $z$-scores for the converters for tests of episodic memory, semantic memory, and executive functioning. TMT-B = Trail Making Test-Part B; $n-2=2$ years before participants' diagnoses converted to Alzheimer's disease; $n-1=1$ year before conversion; $n=$ year of conversion. Error bars represent $\pm 1 S E$. 


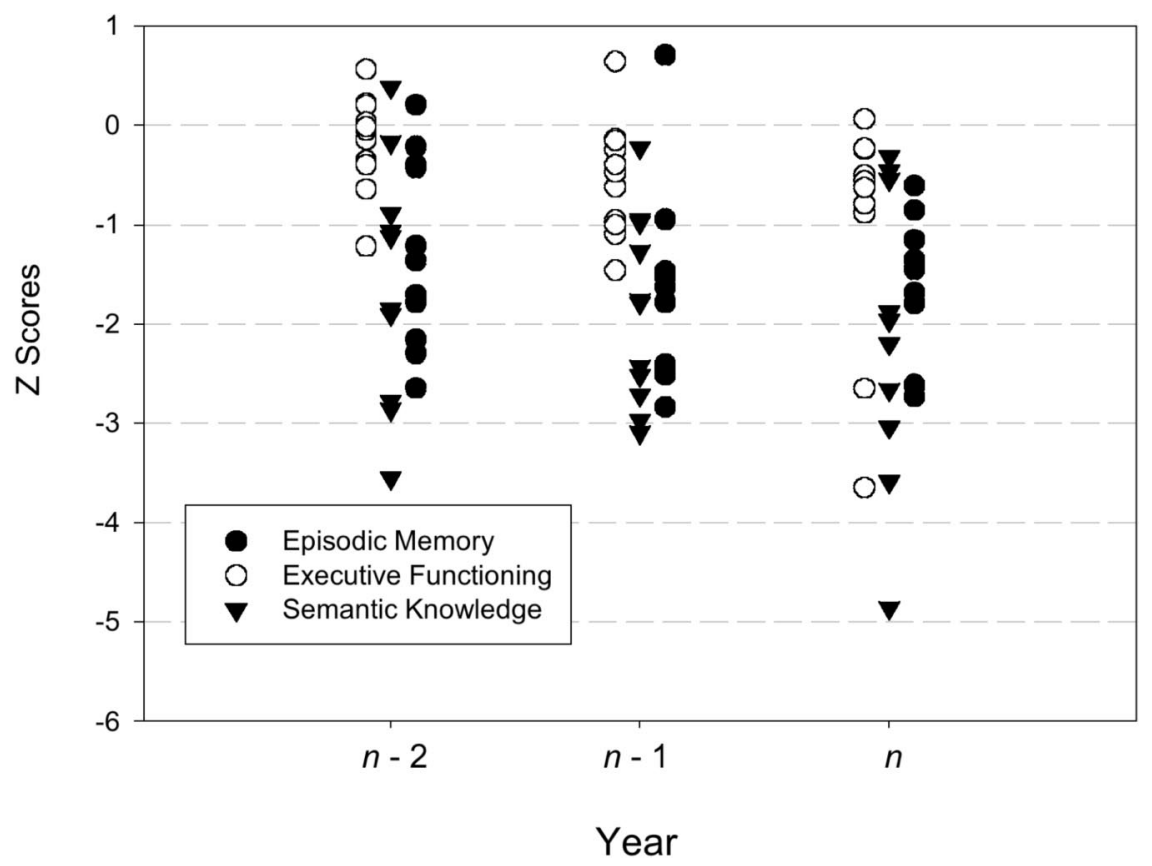

Figure 2. Individual control-referenced $z$-scores for the converters for tests of episodic memory, semantic memory, and executive functioning. $n-2=2$ years before participants' diagnoses converted to Alzheimer's disease; $n-1=1$ year before conversion; $n=$ year of conversion.

by year $n$ they appeared to be a clearly distinct subset of the 11 CONVs. This is visually apparent in the aggregate measure of executive functioning for year $n$ shown in Figure 2, where the scores for these two CONVs fall far below the scores for the rest of the group.

\section{General Discussion}

Our investigation of the neuropsychological profile of 11 individuals with preclinical AD yielded conclusions that differ somewhat from prevailing views about the impact of the disease in the years preceding a nonnormal diagnosis. In a detailed review of the literature concerned with preclinical AD, Backman et al. (2004, 2005), for example, argued that the cognitive decline in the 2 or 3 years preceding a nonnormal diagnosis is largely nonspecific. However, they reviewed methodologically diverse studies, many of which did not follow the design guidelines that characterized our study. Our study analyzed the performance of individuals who, initially, had a normal diagnosis but who eventually converted to a diagnosis of probable $\mathrm{AD}$, and we compared their performance to that of a carefully matched control group. The results suggest that cognitive functions that are thought to be subserved by the medial and lateral temporal lobes (episodic memory and semantic knowledge, respectively) are substantially more impaired than the cognitive functions that are thought to be subserved by the frontal lobes (executive functioning). To be sure, our results also suggest that a small subset of our CONVs (2 of the 11) may have had frontal impairment even though, in the aggregate, the CONV group was relatively unimpaired in this regard. The idea that a subset of preclinical AD patients suffered from frontal impairment is consistent with previous findings (Johnson, Head, Kim, Starr, \&
Cotman, 1999; Waltz et al., 2004). By and large, however, our preclinical CONVs appear to have comparatively intact frontal lobe functioning.

The sample size in our investigation is small because of the rigorous inclusion criteria. To be included, subjects who were initially judged to be cognitively normal had to eventually be diagnosed with probable $\mathrm{AD}$, and complete neuropsychological profiles had to be available as well. The small sample size precludes us from making any strong claims about nonsignificant results (which could be due merely to a lack of power), but it does not argue against claims based on statistically significant results. Our primary claims are based on statistically significant differences in the degree of deficit exhibited by CONVs relative to that of matched controls on tests of episodic memory, semantic knowledge, and executive functioning.

The control participants who are recruited to serve in our ongoing longitudinal investigation of AD (some of whom converted to probable $\mathrm{AD}$ ) are a relatively high-functioning group. For example, as shown in Table 1, the average years of education for the CONV and NC subjects was 16.9. Also, as indicated earlier, the control group scored approximately one standard deviation above age-corrected norms on the Logical Memory Test. Table 4 shows the performance of patients and controls relative to standard norms for the Logical Memory Test, the CVLT (cued recall and free recall), and the TMT-A and TMT-B. In almost every case, the control subjects fell well above normative performance for their age group. As such, our conclusions apply to relatively highfunctioning individuals with characteristics similar to those of our control subjects. Also, in one sense, it is not accurate to portray the CONV group as "normal" in years $n-1$ and $n-2$ because, 
Table 4

Means (and SDs) of the CONV and NC Norms for Cued Recall and Free Recall Tests of the California Verbal Learning Test

\begin{tabular}{|c|c|c|c|c|c|c|}
\hline \multirow[b]{2}{*}{ Measure } & \multicolumn{2}{|c|}{$n-2$} & \multicolumn{2}{|c|}{$n-1$} & \multicolumn{2}{|c|}{$n$} \\
\hline & $\mathrm{NC}$ & CONV & $\mathrm{NC}$ & CONV & $\mathrm{NC}$ & CONV \\
\hline Cued recall & $1.40(1.6)$ & $0.02(0.7)$ & $1.35(1.4)$ & $-1.07(1.5)$ & $1.26(1.4)$ & $-1.14(1.7)$ \\
\hline Free recall & $1.26(1.4)$ & $-0.25(1.0)$ & $1.33(1.4)$ & $-1.10(1.2)$ & $1.26(1.0)$ & $-1.04(1.3)$ \\
\hline Logical memory norms & $13.3(3.2)$ & $10.3(2.4)$ & $12.8(2.8)$ & $10.45(3.53)$ & $13.6(3.9)$ & $8.8(3.1)$ \\
\hline TMT-A & $-0.17(1.2)$ & $-0.42(0.8)$ & $0.11(0.8)$ & $-0.43(0.8)$ & $0.3(0.9)$ & $-0.87(0.5)$ \\
\hline TMT-B & $0.52(0.9)$ & $-0.28(0.6)$ & $0.56(0.8)$ & $-0.57(1.0)$ & $0.59(0.9)$ & $-0.96(1.2)$ \\
\hline
\end{tabular}

Note. Normative data for the California Verbal Learning Test subscales are from Paolo, Troster, and Ryan (1997). Normative data for the Trail Making Test-Parts A and B are from Ivnik et al. (1992). CONV = Participants whose diagnosis converted to Alzheimer's disease during the study. NC $=$ normal controls; $n-2=2$ years before conversion; $n-1=1$ year before conversion; $n=$ year of conversion; TMT-A = Trail Making Test-Part A; TMT-B = Trail Making Test-Part B.

although they are not necessarily impaired with respect to standard norms, they are also not normal with respect to the high-functioning matched control group. Indeed, the CONV group scored more than 1.5 standard deviations below the matched control group on five different tests in year $n-1$ (see Table 3 ). Thus, the preclinical years $(n-1$ and $n-2)$ are best thought of as the 2 years preceding detectable impairment using standard diagnostic tests, not the 2 years preceding a significant decline from a (relatively high) baseline level of functioning.

Despite these considerations, our findings still support our claim that impairment before the onset of $\mathrm{AD}$ is most prominent in the domains of episodic and semantic memory. In year $n-2$, for example, only one of the CONVs fell more than one standard deviation below the mean of the controls for the executive functioning measure (as shown in Figure 2), yet more than half of the CONVs fell more than one standard deviation below the mean of the controls for the episodic and semantic measures.

The conclusions presented above fit with some previous studies on the progression of neurofibrillary tangles in AD. Although controversy exists over whether it is neurofibrillary tangles, amyloid deposits, or neuronal and synaptic loss that constitute the primary neuropathologic process that underlies the disease, neurofibrillary tangle densities in the medial temporal lobes do correlate with clinical evidence of memory impairment (Giannakopoulos et al., 2003). Delacourte et al. (1999) conducted a detailed postmortem analysis of neurofibrillary degeneration in the brains of 130 patients of different ages and differing degrees of dementia (ranging from nondemented to severe AD). On the basis of their findings, they proposed that neurofibrillary degeneration progresses through a 10-stage process, beginning in the entorhinal cortex and then proceeding to affect the hippocampus, the anterior temporal cortex, the inferior temporal cortex, and the middle temporal cortex. Only later do the frontal regions become involved (cf. Fewster, Griffin-Brooks, MacGregor, Ojaivo-Rose, \& Ball, 1991). The authors note that up through Stage 6 individuals could be symptom free, whereas all of those above Stage 7 exhibited dementia. Thus, it seems reasonable to suppose that most of our preclinical AD patients would fall within Stages 1 through 6 (during which time frontal degeneration is not apparent). Viewed in this light, our findings suggest that the progression of cognitive deficits corresponds with the progression of neurofibrillary degeneration.

Imaging studies also offer some support for the idea that frontal impairment appears after temporal lobe impairment. Using positron emission topography, Bradley et al. (2002) found that as $\mathrm{AD}$ progresses from the preclinical to clinical stages, there is an evolution in brain regions that show reduced perfusion. The deficits begin in the medial temporal lobes, the subcallosal region, and the posterior cingulate region. Temporal-parietal perfusion deficits appear next, followed by frontal deficits later in the disease progression. Similarly, Fox et al. (2001) studied 4 preclinical AD patients from families with the early-onset form of the disease using magnetic resonance imaging over a period of 5 to 8 years. The patients were initially symptom-free, but they all became symptomatic during the course of the study. Their results showed that the patients exhibited evidence of cerebral atrophy before they fulfilled the clinical criteria for $\mathrm{AD}$ (i.e., during the preclinical stages of the illness). The observed losses mainly involved the medial temporal lobe, the inferolateral temporal lobe, the parietal lobe, and the posterior cingulate gyrus.

\section{Conclusion}

In summary, our findings suggest that preclinical AD primarily affects the functioning of the temporal lobes (both medial and lateral) with lesser effects on the functioning of the frontal lobes. Performance on measures assessing all these areas declines significantly during the preclinical years, and even performance on the frontal lobe measures suggests substantial impairment by the time a nonnormal diagnosis is made. Still, what is striking about our findings is that the tests most sensitive to the integrity of the temporal lobes suggested greater impairment, relative to matched control subjects, than did the tests most sensitive to the integrity of the frontal lobes. Conceivably, the tests of frontal integrity may simply be relatively insensitive to damage in that area of the brain, whereas the temporal lobe measures may be more sensitive to the presence of temporal lobe damage. Thus, it is possible that more sensitive neuropsychological tests (e.g., the Stroop task) would suggest that the frontal lobes are more impaired in preclinical AD than our data imply.

It is also important to note that although we see a distinct pattern of cognitive decline over the 2 years prior to conversion, it is likely that this preclinical course varies across individuals. These results suggest that in the typical case, episodic memory and semantic knowledge are the most impaired, with relatively less impairment in frontal lobe functions. However, we are limited in understanding the individual profiles, and it is pos- 
sible that our CONV group included different subtypes of AD (Petersen, 2004). For the moment, though, our findings suggest that the frontal lobes are less involved than are the temporal lobes in preclinical $\mathrm{AD}$.

\section{References}

Albert, M. S., Moss, M. B., Tanzi, R., \& Jones, K. (2001). Preclinical prediction of $\mathrm{AD}$ using neuropsychological tests. Journal of the International Neuropsychological Society, 7, 631-639.

Anderson, S. W., Damasio, H., Jones, R. D., \& Tranel, D. (1991). Wisconsin Card Sorting Test performance as a measure of frontal lobe damage. Journal of Clinical and Experimental Neuropsychology, 13, 909-922.

Backman, L., Jones, S., Berger, A. K., Laukka, E. J., \& Small, B. J. (2004). Multiple cognitive deficits during the transition to Alzheimer's disease. Journal of Internal Medicine, 256(3), 195-204.

Backman, L., Jones, S., Berger, A. K., Laukka, E. J., \& Small, B. J. (2005). Cognitive impairment in preclinical Alzheimer's disease: A meta-analysis. Neuropsychology, 19, 520-531.

Berman, K. F., Ostrem, J. L., Randolph, C., Gold, J., Goldberg, T. E., Coppola, R., et al. (1995). Physiological activation of a cortical network during performance of the Wisconsin Card Sorting Test: A positron emission tomography study. Neuropsychologia, 33(8), 1027-1046.

Braak, H., \& Braak, E. (1996). Evolution of the neuropathology of Alzheimer's disease. Acta Neurologica Scandinavica Supplementum, 165, $3-12$.

Bradley, K. M., O’Sullivan, V. T., Soper, N. D., Nagy, Z., King, E. M., Smith, A. D., et al. (2002). Cerebral perfusion SPET correlated with Braak pathological stage in Alzheimer's disease. Brain, 125(8), 17721781.

Butters, N., Granholm, E., Salmon, D. P., Grant, I., \& Wolfe, J. (1987). Episodic and semantic memory: A comparison of amnestic and demented patients. Journal of Clinical and Experimental Neuropsychology, 9, 479-497.

Delacourte, A., David, J. P., Sergeant, N., Buee, L., Wattez, A., Vermersch, P., et al. (1999). The biochemical pathway of neurofibrillary degeneration in aging and Alzheimer's disease. Neurology, 52(6), 1158-1165.

Delis, D. C., Kramer, J. H., Kaplan, E., \& Ober, B. A. (1987). The California Verbal Learning Test. New York: Psychological Corporation.

Demakis, G. J. (2003). A meta-analytic review of the sensitivity of the Wisconsin Card Sorting Test to frontal and lateralized frontal brain damage. Neuropsychology, 17(2), 255-264.

Demakis, G. J. (2004). Frontal lobe damage and tests of executive processing: A meta-analysis of the category test, Stroop test, and Trail Making Test. Journal of Clinical and Experimental Neuropsychology, 26(3), 441-450.

Fewster, P. H., Griffin-Brooks, S., MacGregor, J., Ojaivo-Rose, E., \& Ball, A. (1991). A topographical pathway by which histopathological lesions disseminate through the brain of patients with Alzheimer's disease. Dementia, 2, 121-132.

Folstein, M. F., Folstein, S. E., \& McHugh, P. R. (1975). Mini-mental state: A practical method for grading the cognitive state of patients for the clinician. Journal of Psychiatric Research, 12, 189-198.

Fox, N. C., Crum, W. R., Scahill, R. I., Stevens, J. M., Janssen, J. C., \& Rossor, M. N. (2001). Imaging of onset and progression of Alzheimer's disease with voxel-compression mapping of serial magnetic resonance images. The Lancet, 358(9, 277), 201-205.
Galasko, D., Hansen, L. A., Katzman, R., Wiederholt, W., Masliah, E., Terry, R., et al. (1994). Clinical-neuropathological correlations in Alzheimer's disease and related dementias. Archives of Neurology, 51(9), $888-895$.

Gearing, M., Mirra, S. S., Hedreen, J. C., Sumi, S. M., Hansen, L. A., \& Heyman, A. (1995). The Consortium to Establish a Registry for Alzheimer's Disease (CERAD). Part X. Neuropathology confirmation of the clinical diagnosis of Alzheimer's disease. Neurology, 45(3, Pt. 1), 461466.

Giannakopoulos, P., Herrmann, F. R., Bussiere, T., Bouras, C., Kovari, E., Perl, D. P., et al. (2003). Tangle and neuron numbers, but not amyloid load, predict cognitive status in Alzheimer's disease. Neurology, 60(9), 1495-1500.

Heaton, R. K., Chelune, G. J., Talley, J. L., Kay, G. C., \& Curtis, G. (1993). Wisconsin Card Sorting Test manual: Revised and expanded. Odessa, FL: Psychological Assessment Resources.

Henry, J. D., Crawford, J. R., \& Phillips, L. H. (2004). Verbal fluency performance in dementia of the Alzheimer's type: A meta-analysis. Neuropsychologia, 42(9), 1212-1222.

Ivnik, R. J., Malec, J. F., Smith, G. E., Tangalos, E. G., Peterson, R. C., Kokmen, E., \& Kurland, L. T. (1992). Mayo's older American normative studies: WAIS-R norms for ages 56-97. The Clinical Neuropsychologist, 6, 1-30.

Johnson, J. K., Head, E., Kim, R., Starr, A., \& Cotman, C. W. (1999). Clinical and pathological evidence for a frontal variant of Alzheimer's disease. Archives of Neurology, 56, 1233-1239.

Kaplan, E., Goodglass, H., \& Weintraub, S. (1983). Boston Naming Test. Philadelphia: Lea \& Fibiger.

Lange, K. L., Bondi, M. W., Salmon, D. P., Galasko, D., Delis, D. C., Thomas, R. G., et al. (2002). Decline in verbal memory during preclinical Alzheimer's disease: Examination of the effect of APOE genotype. Journal of the International Neuropsychological Society, 8(7), 943-955.

Levy, D. A., Bayley, P. J., \& Squire, L. R. (2004). The anatomy of semantic knowledge: Medial vs. lateral temporal lobe. Proceedings of the National Academy of Sciences USA, 101(17), 6710-6715.

Lineweaver, T. T., Bondi, M. W., Thomas, R. G., \& Salmon, D. P. (1999). A normative study of Nelson's (1976) modified version of the Wisconsin Card Sorting Test in healthy older adults. Clinical Neuropsychology, 13(3), 328-347.

Mattis, S. (1976). Mental status examination for organic mental syndrome in the elderly patient. In L. Bellack \& T. B. Karasu (Eds.), Geriatric Psychiatry (pp. 77-122). New York: Grune \& Stratton.

McKhann, G., Drachman, D., Folstein, M., Katzman, R., Price, D., \& Stadlan, E. M. (1984). Clinical diagnosis of Alzheimer's disease: Report of the NINCDS-ADRDA Work Group under the auspices of Department of Health and Human Services Task Force on Alzheimer's Disease. Neurology, 34(7), 939-944.

Monsch, A. U., Bondi, M. W., Butters, N., Paulsen, J. S., Salmon, D. P., Brugger, P., \& Swenson, M. R. (1994). A comparison of category and letter fluency in Alzheimer's disease and Huntington's disease. Neuropsychology, 8(1), 25-30.

Nelson, H. E. (1976). A modified sorting test sensitive to frontal lobe deficits. Cortex, 12, 313-324.

Paolo, A. M., Troster, A. I., \& Ryan, J. J. (1997). California Verbal Learning Test: Normative data for the elderly. Journal of Clinical and Experimental Neuropsychology, 19, 220-234.

Petersen, R. C. (2004). Mild cognitive impairment as a diagnostic entity. Journal of Internal Medicine, 256(3), 183-194.

Reitan, R. (1958). Validity of the Trail Making Test as an indicator of organic brain disease. Perceptual and Psychomotor Skills, 8, 271276. 
Salmon, D. P., Thomas, R. G., Pay, M. M., Booth, A., Hofstetter, C. R., Thal, L. J., \& Katzman, R. (2002). Alzheimer's disease can be accurately diagnosed in very mildly impaired individuals. Neurology, 59(7), 10221028.

Saxton, J., Lopez, O. L., Ratcliff, G., Dulberg, C., Fried, L. P., Carlson, M. C., et al. (2004). Preclinical Alzheimer's disease: Neuropsychological test performance 1.5 to 8 years prior to onset. Neurology, 63, 2341-2347.

Small, B. J., Mobly, J. L., Laukka, E. J., Jones, S., \& Backman, L. (2003). Cognitive deficits in preclinical Alzheimer's disease. Acta Neurologica Scandinavica Supplementum, 179, 29-33.

Squire, L. R., \& Zola, S. M. (1996). Structure and function of declarative and nondeclarative memory systems. Proceedings of the National Academy of Sciences, USA, 93(24), 13515-13522.

Waltz, J. A., Knowlton, B. J., Holyoak, K. J., Boone, K. B., BackMadruga, C., McPherson, S., et al. (2004). Relational integration and executive function in Alzheimer's disease. Neuropsychology, 18(2), 296-305.

Wechsler, D. (1945). A standardized memory scale for clinical use. Journal of Psychology, 19, 87-95.

Wixted, J. T., \& Squire, L. R. (2004). Recall and recognition are equally impaired in patients with selective hippocampal damage. Cognitive, Affective, \& Behavioral Neuroscience, 4(1), 58-66.

Yonelinas, A. P., Kroll, N. E., Quamme, J. R., Lazzara, M. M., Sauve, M. J., Widaman, K. F., et al. (2002). Effects of extensive temporal lobe damage or mild hypoxia on recollection and familiarity. Nature Neuroscience, 5(11), 1236-1241.

Received August 21, 2006 Revision received May 18, 2007 Accepted May 30, 2007

\section{American Psychological Association SubSCRIPTION Claims INFORMATION}

Today's Date:

We provide this form to assist members, institutions, and nonmember individuals with any subscription problems. With the appropriate information we can begin a resolution. If you use the services of an agent, please do NOT duplicate claims through them and directly to us. PLEASE PRINT CLEARLY AND IN INK IF POSSIBLE.

PRINT FULL NAME OR KEY NAME OF INSTITUTION

ADDRESS

$\overline{\text { CITY STATE/COUNTRY }}$ ZIP

YOUR NAME AND PHONE NUMBER

TITLE
MEMBER OR CUSTOMER NUMBER (MAY BE FOUND ON ANY PAST ISSUE LABEL)

DATE YOUR ORDER WAS MAIIED (OR PHONED)

_ PREPAID ___ CHECK _ CHARGE CHECK/CARD CLEARED DATE:

(If possible, send a copy, front and back, of your cancelled check to help us in our research of your claim.)

ISSUES: MISSLNG DAMAGED
NUMBER OR MONTH

Thank you. Once a claim is received and resolved, delivery of replacement issues routinely takes 4-6 weeks.

(TO BE FILLED OUT BY APA STAFF)

DATE RECEIVED:

ACTION TAKEN:

STAFF NAME:
DATE OF ACTION:

INV. NO. \& DATE:

LABEL NO. \& DATE:

Send this form to APA Subscription Claims, 750 First Street, NE, Washington, DC 20002-4242

PLEASE DO NOT REMOVE. A PHOTOCOPY MAY BE USED. 